\title{
The relationship between frontal sinus morphology and skeletal maturation
}

\author{
S.K. Buyuk ${ }^{1}$, H. Simsek², A. Karaman ${ }^{1}$ \\ ${ }^{1}$ Department of Orthodontics, Faculty of Dentisty, Ordu University, Ordu, Turkey \\ ${ }^{2}$ Department of Paediatric Dentistry, Faculty of Dentistry, Ordu University, Ordu, Turkey
}

[Received: 8 October 2017; Accepted: 29 November 2017]

\begin{abstract}
Background: The aim of this study is to evaluate the relationship between frontal sinus morphology and hand-wrist bone maturation by using postero-anterior (PA) cephalometric radiographs.

Materials and methods: The study sample consisted of 220 patients divided into 11 groups based on the hand-wrist radiographs. The right and left maximum height, width and area of the frontal sinus parameters were measured in PA cephalometric radiographs of 220 subjects aged 8-18 years. The hand-wrist skeletal maturation stages were evaluated on the hand-wrist radiographs using the method of Fishman. The Kendall tau- $b$ values were analysed to evaluate the correlation between the hand-wrist skeletal maturation stages and the frontal sinus parameters.
\end{abstract}

Results: The right and left frontal sinus areas and widths were found to be larger in males than in females $(p<0.05)$. In males, a significant difference was observed in all frontal sinus parameters in different maturation stages $(p<0.001)$, while a statistically significant correlation was found in females between the left frontal sinus area, right frontal sinus height, right frontal sinus width and different maturation stages $(p<0.05)$.

Conclusions: The relationship between frontal sinus dimensions obtained from PA cephalometric radiographs and hand-wrist maturation stages suggests that frontal sinuses can be used in determining growth and development. (Folia Morphol 2018; 77, 3: 503-508)

Key words: frontal sinus dimension, growth spurt, hand-wrist radiographs

\section{INTRODUCTION}

In orthodontic treatment planning, it is important to know the growth potential of craniofacial region. The stage of growth spurt in the adolescent or preadolescent period has a significant effect on both planning of orthodontic treatment and maintenance of the results obtained after orthodontic treatment [8]. Different parameters are used to describe pubertal growth in children. Some of the parameters that are used in determining pubertal growth spurt by assessing growth and development in adolescence are chronological age, pubic hair appearance in males and females, menstruation onset in females, radiographic evaluation of bone maturation stages and evaluation of tooth calcification stages $[8,16]$.

The hand-wrist radiographs are frequently used in orthodontics and paediatric dentistry in order to evaluate skeletal maturity. The hand-wrist radiographs are

Address for correspondence: Dr. H. Simsek, Ordu University, Faculty of Dentistry, Department of Paediatric Dentistry, Turkey, e-mail: dr.huseyinsimsek@gmail.com 
preferred because hand-wrist bones can be examined in a single radiographic image and constitute a region that can be radiographically well analysed $[7,8]$. It is also considered to be the most reliable method for assessing skeletal maturity because of the presence of a large number of bones and the opportunity it provides to observe ossification in a wide time interval, as the ossification time of the epiphysial and diaphysial parts of the finger bones differs $[2,6]$.

The frontal sinuses are paranasal sinuses surrounding the nasal cavity, which are analysed during routine examinations before orthodontic treatment and among structures visible in lateral and posteroanterior (PA) cephalometric radiographs [3]. Unlike other paranasal sinuses, the frontal sinuses are not visible at birth and can be radiographically seen at $4-6$ years $[5,11,17]$. The height, width, and volume of frontal sinuses continue to increase until the age of 20 [17]. Studies demonstrated that frontal sinuses develop simultaneously with growth. Researchers reported that the size of frontal sinuses is related to the growth pattern and grade of craniofacial structures $[14,15]$.

Although there are a limited number of studies that evaluated dimensions of frontal sinuses and skeletal maturity by using lateral cephalometric radiographs $[13,15]$, we could not find any study that determined a relationship between the size of frontal sinuses and hand-wrist bone maturation by using PA cephalometric radiographs. The aim of this study is to evaluate the relationship between frontal sinus morphology and hand-wrist bone maturation by using PA cephalometric radiographs.

\section{MATERIALS AND METHODS}

This study was designed as a retrospective and cross-sectional research study. The samples were derived from the pretreatment hand-wrist radiographs and PA cephalometric radiographs of orthodontic patients visiting our dental clinics at Ordu University, Faculty of Dentistry in Ordu University, Turkey. The sample size was calculated based on a power analysis using G*Power Software version 3.1.9.2 (Universität Düsseldorf, Germany) for a right frontal sinus area at alpha error probability of 0.05 and a power of $95 \%$ (effect size $=1.0095429$ ) [3]. The power analysis showed that 44 samples were required, totally. However, our total sample size was 220 subjects, and we divided into uniformly-sized 11 group and 20 subjects in each developmental stage. Skeletal maturation

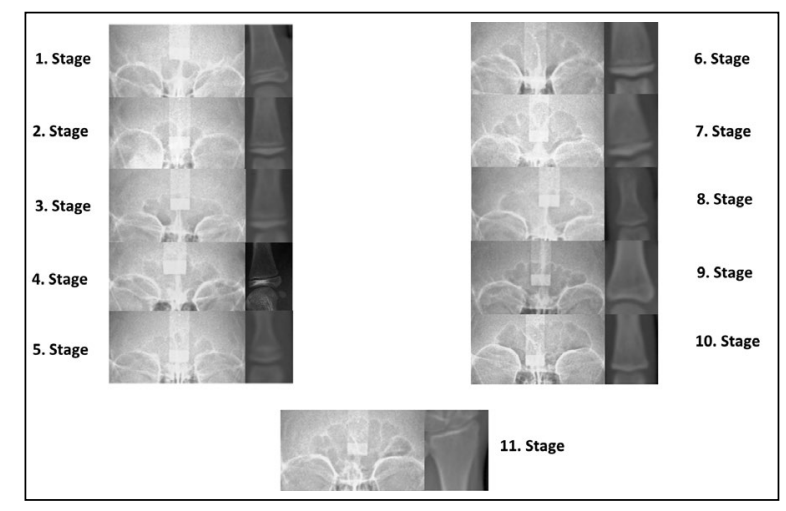

Figure 1. Different postero-anterior cephalometric radiographs correlated with the hand-wrist skeletal maturity by using Fishman method.

stage of each hand-wrist radiograph was determined according to the method described by Fishman [6].

The patients within the age range of 8-18 years were included in this study; they were at different stages of skeletal development. Patient without systemic disease and having normal overall growth and development were considered for the study. Patient with low-quality hand-wrist radiographs and PA cephalometric radiographs; those who had undergone previous orthodontic treatment or permanent tooth extraction; subjects with any developmental endocrine or nutritional disorders; and subjects with history of injury or trauma to the face or hand and wrist regions have been excluded from the study. Also, patient with sinus-related trauma, pathologies or surgery involving the frontal sinus were not included in the study.

The hand-wrist radiographs analysis was evaluated according to the Fishman skeletal maturation stages. Patients' skeletal ages were determined by the same researcher (A.K.) by evaluating hand-wrist radiographs using the Fishman method. For every subject, the frontal sinus dimension parameters obtained in the PA radiograph was correlated with the hand-wrist skeletal maturity obtained with Fishman method in the hand-wrist radiograph (Fig. 1).

All frontal sinus parameters were determined in $P A$ radiographs. For determining frontal sinus dimensions, the $Z$ line was considered as both the upper border of orbital cavity and the lower border of the frontal sinus. The right and left area of the frontal sinus, the right and left height, the right and left width parameters of the region above the bottom line of the frontal sinus were taken as the measurements. 


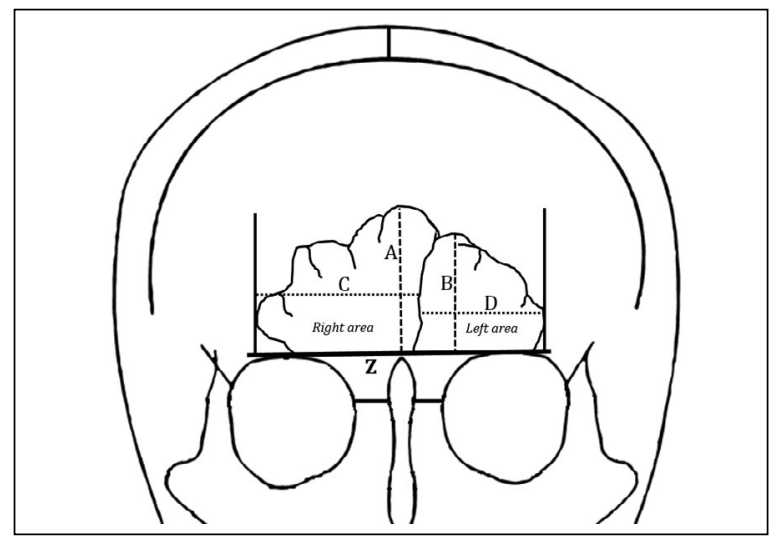

Figure 2. Diagrammatic measurements of the frontal sinus dimensions. A, B, C, D, Z abbreviations - see text.

The separation of the frontal sinuses into the right and left sides are based on the main midline septum of the frontal sinus. The difference between the lowest and highest points on the right and left sides of the frontal sinus represented the heights specified as ' $A$ ' and ' $B$ '. The widths specified as ' $C$ ' and ' $D$ ', indicated the width difference between the medial and lateral points on the right and left sides of the frontal sinus (Fig. 2). All PA cephalograms were obtained by the same cephalometric film device (Kodak 8000C Digital Panoramic and Cephalometric System, Cephalostat, Corestream Health Inc, France) and the patients' Frankfurt plane parallel to the ground plane. Linear measurements of the frontal sinus were done by using a cephalometric software programme. Areas of the frontal sinus dimensions were obtained using Image J analysis software by calibrating (NIH Image, Bethesda, USA).

\section{Statistical analysis}

All measurements were statistically analysed by using SPSS (SPSS for Windows version 20.0; SPSS Inc, Chicago, IL, USA) programme. After performing the normal distribution test, non-parametric tests were applied to the parameters with non-normal distributions, while parametric tests were performed to the parameters having a normal distribution. In intergender comparisons, independent t-test and MannWhitney $\mathrm{U}$ test were used. Comparisons between frontal sinus dimension measurements among the different hand-wrist skeletal stages were performed using the Kruskal-Wallis test. Correlation between the hand-wrist skeletal maturational stages and frontal sinus parameters were based on the Kendall tau-b correlation coefficient in both sexes. In all tests, values of $p<0.05$ were considered statistically significant.

In order to determine intra-observer reliability, 44 PA cephalometric and hand-wrist radiographs from each group were reevaluated by the same observer 4 weeks after the first assessment. Intra-class correlation coefficient and kappa statistics were used to evaluate intra-observer reliability.

\section{RESULTS}

The intra-class correlation coefficients for the frontal sinus measurements were $>0.990$, and the intra-observer kappa statistic was 1.00 , confirming the measurement reliability. The height, width, and surface area comparisons of right and left frontal sinuses are given in Table 1 . The right and left frontal sinus areas and widths were found to be larger in males than in females. And this difference is statistically significant $(p<0.05)$. However, the heights of both right and left frontal sinuses did not differ between sexes $(p>0.05)$.

In males, a significant difference was observed in all frontal sinus parameters in different hand-wrist maturation stages, while a statistically significant correlation was found in females between the left frontal sinus area, right frontal sinus height, right frontal sinus width and different hand-wrist maturation stages (Table 2).

Statistically significant differences were found in all frontal sinus parameters in different hand-wrist maturational stages in males $(p<0.001)$, whereas statistically significant differences were found in right and left frontal sinus areas and right and left frontal sinus heights in females $(p<0.05)$ (Table 3 ).

\section{DISCUSSION}

The frontal sinuses, which are located between the external and internal cortical layers of the frontal bone, are anatomically called pneumatic cavities [5]. Frontal sinuses, which are not visible radiographically at birth, begin to appear from 3 years and 3 months in males and 4 years and 6 months in females [1]. The size of frontal sinuses increases with age and consequently continues to grow until the age of 20 years [12]. In our study, the area, height and width of frontal sinuses were assessed in both sexes according to hand-wrist developmental stages at different age groups. In addition, like the study by Mahmood et al. [13], individuals over 8 years of age were included in our study. 
Table 1. Comparison of frontal sinus measurements between sexes

\begin{tabular}{|c|c|c|c|c|c|c|c|}
\hline \multirow[t]{2}{*}{ Parameters } & \multicolumn{3}{|c|}{ Male } & \multicolumn{3}{|c|}{ Female } & \multirow[t]{2}{*}{$\mathbf{p a}$} \\
\hline & $25^{\text {th }}$ percentile & Median & $75^{\text {th }}$ percentile & $25^{\text {th }}$ percentile & Median & $75^{\text {th }}$ percentile & \\
\hline Right frontal sinus area $\left[\mathrm{cm}^{2}\right]$ & 1.43 & 2.45 & 3.47 & 1.25 & 1.84 & 2.57 & 0.011 \\
\hline Left frontal sinus area $\left[\mathrm{cm}^{2}\right]$ & 1.35 & 2.54 & 3.84 & 1.27 & 2.02 & 2.69 & 0.022 \\
\hline Right frontal sinus height [mm] & 10.53 & 15.85 & 19.70 & 10.53 & 14.20 & 16.90 & 0.097 \\
\hline \multirow[t]{2}{*}{ Left frontal sinus height [mm] } & 10.65 & 15.10 & 19.68 & 10.83 & 13.70 & 17.90 & 0.300 \\
\hline & Mean & SD & SE & Mean & SS & SE & $\mathbf{P b}$ \\
\hline Right frontal sinus width [mm] & 27.40 & 7.53 & 0.67 & 24.75 & 6.49 & 0.68 & 0.007 \\
\hline Left frontal sinus width [mm] & 27.42 & 7.93 & 0.70 & 25.10 & 7.10 & 0.74 & 0.026 \\
\hline
\end{tabular}

aResults of Mann Whitney $\mathrm{U}$ test; ${ }^{b}$ Results of independent $t$ test; SD — standard deviation; SE — standard error

Table 2. Correlation between frontal sinus measurements and hand-wrist skeletal maturational stage

\begin{tabular}{lccc}
\hline Parameters & Sex & Correlation coefficient $^{\text {a }}$ & P \\
\hline Right frontal sinus area & Male & 0.377 & $<0.001$ \\
& Female & 0.140 & 0.059 \\
Left frontal sinus area & Male & 0.436 & $<0.001$ \\
& Female & 0.202 & 0.006 \\
Right frontal sinus height & Male & 0.333 & $<0.001$ \\
& Female & 0.168 & 0.024 \\
Left frontal sinus height & Male & 0.387 & $<0.001$ \\
& Female & 0.127 & 0.086 \\
Right frontal sinus width & Male & 0.315 & $<0.001$ \\
& Female & 0.202 & 0.006 \\
Left frontal sinus width & Male & 0.347 & $<0.001$ \\
\hline
\end{tabular}

$\mathrm{N}=220$, aKendall's Tau-b correlation values

In our study, right and left frontal sinus areas and right and left frontal sinus heights were greater in males than females. These results are compatible with those of Camargo et al. [3], who evaluated morphologic structure of frontal sinuses by using PA cephalometric radiographs. Moreover, height and width of frontal sinuses were compared with the developmental stages of cervical vertebrae in the study of Mahmood et al. [13]. At the end of the study, they found that the width and height of the frontal sinuses were higher in males than females. In addition, they found a statistically significant relationship between the width and height of frontal sinuses and developmental stages of cervical vertebrae in both sexes. In our study, a statistically significant correlation was observed between all frontal sinus parameters and different hand-wrist maturation stages in males, while here was a statistically significant correlation between left frontal sinus area, right frontal sinus height, right frontal sinus width and different hand-wrist matura- tion stages in females. In our study, the morphological structure of the frontal sinuses was evaluated through PA cephalometric radiographs, while Mahmood et al. [13] performed their evaluations by using lateral cephalometric radiographs. Furthermore, they determined skeletal bone maturation through cervical vertebrae, while we used hand-wrist radiographs in our study as a more accurate method to evaluate skeletal maturation.

Gagliardi et al. [9] examined the relationship between frontal sinus development, body height and ossification stages of hand-wrist bones as a skeletal maturation method. The material of the study consisted of lateral cephalometric radiographs of 31 Aboriginal Australians between 7 and 18 years of age to assess their frontal sinuses, hand-wrist radiographs to assess their skeletal maturation and body heights of these individuals included in the study. As a result of their study, they found a close relationship between frontal sinus development and ossification 


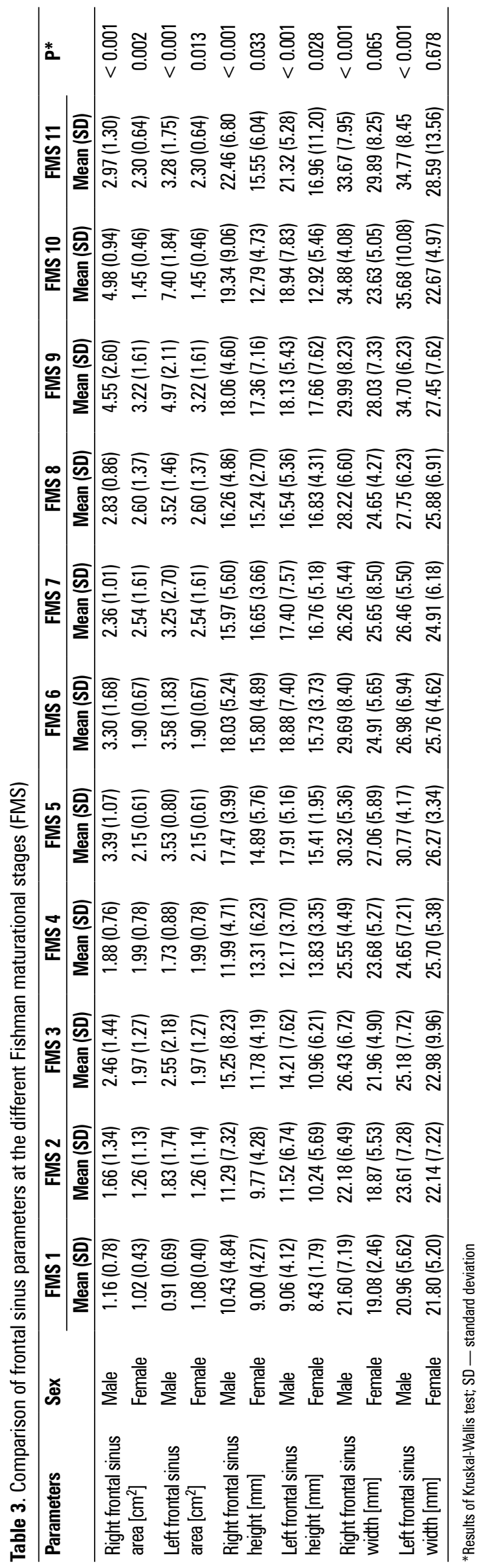

stages of hand-wrist bones. In addition, they reported that the relationship between developmental rapidity of frontal sinuses and ossification of hand-wrist bones may be a used as a useful parameter to predict pubertal growth development stage in orthodonticpaediatric clinical practice. In our study, differently than in this study, frontal sinuses were evaluated using PA cephalometric radiographs. While Gagliardi et al. [9] used Grave and Brown's method [10] on hand-wrist bones to assess skeletal maturation, we used Fishman's method, which is frequently used in orthodontic practice, in our study. Similar to the study by Gagliardi et al. [9], a statistically significant correlation was observed between all frontal sinus parameters and different hand-wrist maturation stages in males, while there was a statistically significant correlation between left frontal sinus area, right frontal sinus height, right frontal sinus width and hand-wrist maturation stages in females.

Similarly to our study, Ruf and Pancherz [15] also found a relationship between developmental stages of frontal sinuses and skeletal maturation of handwrist bones in their study where they suggested that frontal sinuses can be used as skeletal and somatic maturation indicators. However, they evaluated frontal sinuses by using lateral cephalometric radiographs and skeletal maturity using Hagg and Taranger's method on hand-wrist radiographs and they included only male subjects in their studies. Based on the results of their study, they concluded that the size of frontal sinuses could be used to determine pubertal growth and development, as frontal sinuses exhibit a similar pattern after an average of 1.4 years from reaching the peak height. In our study, we think that frontal sinuses with all parameters can be used to determine pubertal growth and development in males and with some parameters in females. Unlike Ruf and Pancherz's study [15], we used anteroposterior cephalometric images in which frontal sinuses were observed in a larger area and Fishman's method to evaluate skeletal maturity from hand-wrist bones.

It is very important to determine pubertal growth and development stages of patients in order to determine an orthodontic treatment plan. It was suggested with this study that PA cephalometric radiographs of frontal sinuses can be used for this purpose. However, our study was a retrospective archival study and conducted on two-dimensional radiographs not to expose patients to extra radiation. In order to support the results of our study, longitudinal studies with 
three-dimensional cone beam computed tomography images are needed.

\section{CONCLUSIONS}

The study results revealed that the right and left frontal sinus areas and widths were larger in males than in females. A significant correlation was found between all frontal sinus parameters and different hand-wrist maturation stages in males, while there was a statistically significant correlation between left frontal sinus area, right frontal sinus height, right frontal sinus width and different hand-wrist maturation stages in females. In conclusion, the relationship between frontal sinus dimensions obtained from PA cephalometric radiographs and hand-wrist maturation stages suggests that frontal sinuses can be used in determining growth and development.

\section{REFERENCES}

1. Brown WA, Molleson TI, Chinn S. Enlargement of the frontal sinus. Ann Hum Biol. 1984; 11(3): 221-226, indexed in Pubmed: 6742771.

2. Camacho-Basallo P, Yáñez-Vico RM, Solano-Reina E, et al. Five radiographic methods for assessing skeletal maturity in a Spanish population: is there a correlation? Acta Odontol Scand. 2017; 75(2): 106-112, doi: 10.1080/00 016357.2016.1265145, indexed in Pubmed: 27934543.

3. Camargo JR, Daruge E, Prado FB, et al. The frontal sinus morphology in radiographs of Brazilian subjects: its forensic importance. Braz J Morphol Sci. 2007; 24: 239-243.

4. Cross DL, McDonald JP. Effect of rapid maxillary expansion on skeletal, dental, and nasal structures: a postero-anterior cephalometric study. Eur J Orthod. 2000; 22(5): 519-528, indexed in Pubmed: 11105408.

5. da Silva RF, Prado FB, Caputo IG, et al. The forensic importance of frontal sinus radiographs. J Forensic Leg Med. 2009; 16(1): 18-23, doi: 10.1016/j.jflm.2008.05.016, indexed in Pubmed: 19061844.

6. Fishman LS. Radiographic evaluation of skeletal maturation. A clinically oriented method based on handwrist films. Angle Orthod. 1982; 52(2): 88-112, doi: 10.1043/0003-3219(1982)052<0088:REOSM>2.0. CO;2, indexed in Pubmed: 6980608.

7. Flores-Mir C, Burgess CA, Champney M, et al. Correlation of skeletal maturation stages determined by cervical vertebrae and hand-wrist evaluations. Angle Orthod. 2006;
76(1): 1-5, doi: 10.1043/0003-3219(2006)076[0001:COS MSD]2.0.CO;2, indexed in Pubmed: 16448261.

8. Flores-Mir C, Nebbe B, Major PW. Use of skeletal maturation based on hand-wrist radiographic analysis as a predictor of facial growth: a systematic review. Angle Orthod. 2004; 74(1): 118-124, doi: 10.1043/0003-3219(2004)074<0118: UOSMBO > 2.0.CO;2, indexed in Pubmed: 15038500.

9. Gagliardi A, Winning T, Kaidonis J, et al. Association of frontal sinus development with somatic and skeletal maturation in Aboriginal Australians: a longitudinal study. Homo. 2004; 55(1-2): 39-52, indexed in Pubmed: 15553267.

10. Grave KC, Brown T. Skeletal ossification and the adolescent growth spurt. Am J Orthod. 1976; 69(6): 611-619, indexed in Pubmed: 179326.

11. Kim DI, Lee UY, Park SO, et al. Identification using frontal sinus by three-dimensional reconstruction from computed tomography. J Forensic Sci. 2013; 58(1): 5-12, doi: 10.1111/j.1556-4029.2012.02185.x, indexed in Pubmed: 22563883.

12. Kjær I, Pallisgaard C, Brock-Jacobsen MT. Frontal sinus dimensions can differ significantly between individuals within a monozygotic twin pair, indicating environmental influence on sinus sizes. Acta Otolaryngol. 2012; 132(9): 988-994, doi: 10.3109/00016489.2012.677064, indexed in Pubmed: 22568667.

13. Mahmood HT, Shaikh A, Fida M. Association between frontal sinus morphology and cervical vertebral maturation for the assessment of skeletal maturity. Am J Orthod Dentofacial Orthop. 2016; 150(4): 637-642, doi: 10.1016/j.ajodo.2016.03.022, indexed in Pubmed: 27692421.

14. Rossouw PE, Lombard CJ, Harris AM. The frontal sinus and mandibular growth prediction. Am J Orthod Dentofacial Orthop. 1991; 100(6): 542-546, doi: 10.1016/08895406(91)70095-E, indexed in Pubmed: 1962608.

15. Ruf S, Pancherz H. Development of the frontal sinus in relation to somatic and skeletal maturity. A cephalometric roentgenographic study at puberty. Eur J Orthod. 1996; 18(5): 491-497, indexed in Pubmed: 8942099.

16. Trakinienè $G$, Smailienė $D$, Kučiauskienè A. Evaluation of skeletal maturity using maxillary canine, mandibular second and third molar calcification stages. Eur J Orthod. 2016; 38(4): 398-403, doi: 10.1093/ejo/cjv051, indexed in Pubmed: 26378083.

17. Yun InS, Kim YO, Lee SK, et al. Three-dimensional computed tomographic analysis of frontal sinus in Asians. J Craniofac Surg. 2011; 22(2): 462-467, doi: 10.1097/ SCS.0b013e3182074367, indexed in Pubmed: 21403576. 